\title{
Perception of undergraduate students in speech-language pathology about speech therapy in the field of deafness
}

\author{
Renata da Silva Gonçalves ${ }^{1}$ \\ https://orcid.org/0000-0002-5387-1104 \\ Maria Cecília Marconi Pinheiro Lima² \\ https://orcid.org/0000-0002-4203-0019 \\ Angélica Bronzatto de Paiva e Silva² \\ https://orcid.org/0000-0001-5181-0650 \\ Maria Elisabete Rodrigues Freire Gasparetto ${ }^{2}$ \\ https://orcid.org/0000-0002-5221-0064
}

Universidade Estadual de Campinas, Curso de Fonoaudiologia, Faculdade de Ciências Médicas, Campinas, São Paulo, Brasil.

Universidade Estadual de Campinas, Faculdade de Ciências Médicas, Departamento de Desenvolvimento Humano e Reabilitação, Campinas, São Paulo, Brasil.

Research support source: Conselho Nacional de Desenvolvimento Científico e Tecnológico - CNPq; Programa Institucional de Bolsas de Iniciação Cientifica - PIBIC.

Conflict of interests: Nonexistent

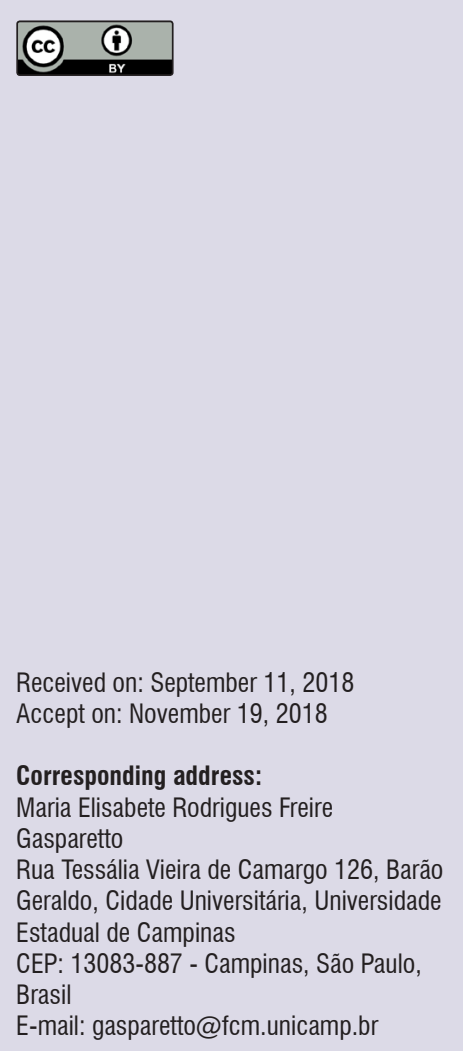

\section{ABSTRACT}

Objective: to know the perception of undergraduate students in speech-language pathology about speech therapies in the area of deafness.

Methods: qualitative research, with data collection accomplished with a questionnaire and a focus group. The sample was accomplished by 14 students enrolled at a speech pathology course.

Results: the results were discussed by means of categories: knowledge, expectations, needs, bond and therapeutic practices. The students presented feelings of anguish in dealing with family expectations, especially regarding to the speech work and the fear for intervening caused by inexperience and necessities of the family. It was also evidenced that the students hoped that family members believed in the potential of the child. There is a need for more practice in Brazilian Sign Language. The support of the family members is crucial for a good therapeutical accompaniment, because when they trust on the student, the kind of therapy, and on the adopted approach, he or she cooperates with the evolution of the therapeutical process, facilitating the bond. When the family believes in the effectiveness of the therapy and the possibility of good results, the child does not miss sessions. Moreover, the bond created with the family and the patient helps the student to see the real difficulties of the child and what should be done to be improved. It was pointed out that the bilingual approach cooperates for the self-recognition of deaf culture and identity.

Conclusion: on students' perception, the experience gained along the stage provided theoretical-practical knowledge to work with the family, recognizing the importance of this to the therapeutic success and to deal with the expectations of both parties.

Keywords: Clinical Clerkship; Speech, Language and Hearing Sciences; Deafness; Education, Higher 


\section{INTRODUCTION}

Speech-Language Pathology is the health science that addresses aspects related to human communication in its different domains and scope. The education of speech-language pathologists aims to provide the professional with the knowledge needed to the skills and abilities required to improve human communication, habilitation and/or rehabilitation therapy and guidance to patients and their families ${ }^{1}$.

Clinic schools are the intersection point between training and professional practice. These schools have social relevance in the health area, since they connect the producer of scientific knowledge, the social demand and the commitment to training. In addition to diversifying the possibilities of action and due to the relevance and representativeness in health care, the work provided in school clinics contributes to a broader training for the students, thus meeting the real needs of the community².

The training of creative professionals with social commitment and interdisciplinary practices is an increasing demand in the labor market. Actions are required to assist in changes in the academic training and in the improvement in the education to train these professionals. In this way, the student must be exposed to theoretical and practical activities that allow evaluation and improvement of their learning ${ }^{3}$.

The complexity of the supervised curricular stage occurs when students use a knowledge that they acquired from specific theoretical content to solve the problems of the population served by them. Thus, the student develops technical and humanistic skills for the profession through orientations and feedback provided by the supervising teacher on their professional and educational development, in order to provide an adequate care; to enhance their theoretical-practical knowledge and to train a critical and reflexive professional, who should be capable of acting within the lived scenario and should be able to meet the social demands ${ }^{4}$.

The Clinical Speech-Language Pathology Practical Training subject is offered in the Speech-Language Pathology Course provided by the institution included in the study. Such subject is provided in the fifth and sixth semesters of the Course and it is divided into two topics: acting with children and adolescents with language delay and acting with deaf children and adolescents. With respect to the care provided to deaf patients, the subject is designed to assess the patient's language, the continuity in the therapeutic follow-up of patients who have already been evaluated, and the analysis of the therapeutic process, including the evolution and prognosis of the deaf child/adolescent from the bilingual perspective. In addition, the subject proposes a group discussion of cases attended by students under the guidance of teachers, including the preparation of therapeutic follow-up reports. The characteristics of the subject are shown in Figure 1 below.

\begin{tabular}{|c|l|}
\hline \multicolumn{2}{|c|}{ Subject: FN509 - Practical Training in Speech-Language Pathology Clinic I } \\
\hline Subject Syllabus & $\begin{array}{l}\text { Initiation of clinical care. The steps of the clinical process: data collection of medical records, bibliographic } \\
\text { survey for case study, presentation, and analysis of complementary tests, therapeutic planning, reporting of } \\
\text { clinical evaluation and therapeutic follow-up. }\end{array}$ \\
\hline Total Workload & 75 hours divided in 19 weeks. \\
\hline Purpose & $\begin{array}{l}\text { To train students in the care of deaf children and their families, providing knowledge on oral and written } \\
\text { language acquisition and development processes, acting with the lip reading and auditory skills. To provide } \\
\text { students with conditions so that they can reflect and act in rehabilitation practice, as appropriate to different } \\
\text { development characteristics of each child. }\end{array}$ \\
\hline Pragmatic Content & $\begin{array}{l}\text { Medical records survey and reading; discussion of fundamental aspects on the interaction with the family; } \\
\text { implementation of speech-language pathology care in oral and written language, auditory skills, lip reading } \\
\text { and communication in general; family guidance on the process of language acquisition, auditory skills, lip } \\
\text { reading and communication in general; Monitoring of hearing aids use and cochlear implant for deaf children; } \\
\text { survey of bibliography that may assist in the case; interdisciplinary discussion of the clinical case in order to } \\
\text { set care criteria; monitoring of developments in the case; development of suitable materials as facilitators of } \\
\text { the interaction between subject/activity/therapist; contact with the school through written reports; daily and } \\
\text { half-yearly reports. }\end{array}$ \\
\hline
\end{tabular}

Figure 1. Subject Syllabus, workload, purpose and pragmatic content of subject 'FN509 - Practical Training in Speech-Language Pathology Clinic l" 
A study with undergraduate students in the health area found that there is a constant tension in the practical training, since these students were not able to separate personal (their feelings) and professional aspects when dealing with patients ${ }^{4}$. Studies that address vocational training contributes to the improvement and efficiency of the care provided to people ${ }^{5}$. With regard to deafness, the student is expected to understand the deaf child/ adolescent as a whole and to understand how they perceive themselves as non-listeners and the obstacles to which they are exposed, i.e. the natural difficulties related to their growth, family conflicts, frustrations, as well as issues related to personal identity and feelings of belonging ${ }^{6}$.

The close relationship between the speech-language pathologist, the family members of the patient and the way each physician acts in relation to their care are closely linked to the clinic design. The bond between the speech-language pathologist and the families and the exchange of information contribute to the development of an essential partnership in care ${ }^{7}$. Knowing and meeting the family with whom you are working is crucial to the success of the therapy and the therapist is required to understand the diversity of families, and must be able to answer that question, gaining personal experience and better effectiveness in working with the family in its most diverse scopes.

The way parents perceive the speech-language intervention, as well as the resources used, the influence of the attitude of the professional, and the quality of the counseling, influences the decisions that will be taken in the relationship between the patient, the family and the student ${ }^{4}$.

Therefore, this study aimed to know the perception of undergraduate students in speech-language pathology about speech therapies in the area of deafness.

\section{METHODS}

The research was approved by the Ethics Research Committee at the University of Campinas UNICAMP, under protocol no. 1.656 .788 and CAAE: 57822516.0.0000.5404. All students signed the Free and Informed Consent Term (FICT).

This study used a focus group and applied a semi-structured instrument, being characterized as a qualitative approach that works with the universe of meanings, motives, values, aspirations, attitudes and beliefs ${ }^{8}$.

The following were set as inclusion criteria: undergraduate students of the $3^{\text {rd }}$ and $4^{\text {th }}$ years of the Speech-Language Pathology Course from the institution, who had served or were serving deaf children and adolescents. Recruitment of students in speech-language pathology course to participate was conducted through an online invitation (e-mail) after drawing from the list of students enrolled in the curricular training offered by this institution.

The researcher was responsible for conducting the focal group ${ }^{9}$ and the triggering questions for the group discussion were as follows: "Perception of undergraduate students in speech-language pathology about the therapy in the field of deafness".

Fourteen students participated in the research and these focal groups were composed and distributed as follows: Focal Group 01 (G1) consisted of seven $3^{\text {rd }}$ year students, which were identified by the letter "A" and Focal Group 02 (G2) consisted of seven $4^{\text {th }}$ year students (that were identified with the letter "B").

In addition to focal group, a self-administered and semi-structured questionnaire was also developed in order to collect and addressing the following topics: "Questions related to supervised training in the field of deafness"; "Perceptions and feelings on the training"; "Needs reported by deaf children/adolescents"; "Facilities and difficulties for patients and their families in a supervised training"; "Perception on interaction with family members and children and/or adolescents and bonding".

Data were collected in the "Gabriel Porto" Center for Studies and Research in Rehabilitation (CEPRE), which is the clinic school of the Speech-Language Pathology undergraduate course. First, the students received and signed the Free and Informed Consent Term and then, after accepting to participate, they answered a selfadministered questionnaire.

In order to ensure the reliability and validity of data collected in the research, the questionnaire was tested with $3 r d$ and 4 th year students of the Speech-Language Pathology Course, to check the interpretation of the questions and to obtain allowances to improve the instrument. Those students who participated in this pre-test with the instrument gave their suggestions and perspectives on the instrument in order to improve it and did not participate in the final data collection.

After the group of participants completed this instrument, the focal group was conducted, which lasted one hour.

The audio and video of focal groups were recorded with a Sony ${ }^{\circledR}$ DSC-W610 camera, for later transcription of speeches. After data collection, a previous survey 
of possible categories was conducted with the results collected through the completion of the questionnaire and the focus group.

The thematic analysis technique was applied, in which the data are grouped by means of categorization and considering the common part existing between them. All questionnaires were reviewed and all discussions were transcribed. The data collected on the answers provided in the questionnaire, observation and participation in the focal groups gave rise to the categories, which refer to aspects of higher frequency according to the opinion of the participants in this research, which are: Knowledge to work in the field of deafness, Expectations of students and families, Specific needs, and Bond and therapeutic practices.

\section{RESULTS}

The results were discussed by means of four categories:

\section{Knowledge to work in the field of deafness}

It may be noticed that most of the students believe that they have both theoretical and practical knowledge to work in the field of deafness (Figure 2). Although they report that they have the required knowledge, students showed an interest in having more specific knowledge in the discussions of the focal group (G1), especially with respect to implantable prostheses (Cochlear Implants) and the Personal Sound Amplification Products (PSAPs). As reported by the participant:

\begin{tabular}{|l|c|}
\hline Knowledge acquisition & $\%$ \\
\hline Yes & 11 \\
\hline No & 3 \\
\hline Knowledge acquired in the first four semesters & Number of citations \\
\hline Theoretical and methodological aspects of working with the family & 6 \\
\hline Brazilian Sign Language (BSL) & 2 \\
\hline Group Dynamics & 1 \\
\hline Hearing Anatomy and Physiology & 1 \\
\hline Knowledge acquired on the 5th and 6th semesters & Number of citations \\
\hline Theoretical aspects of deafness (history and cases) & 5 \\
\hline During supervision & 6 \\
\hline
\end{tabular}

Figure 2. Knowledge to work in the field of deafness

A1: "I believe I have knowledge, but not enough. Patients' families have many questions and have high expectations and I do not always know what to answer or how to better answer their questions. As I do not master the prosthesis or implant issues, I also face problems."

The G2 reported that the theoretical deafness and family subjects that are offered in the first years of the undergraduate course, prior to the practical training, assisted with a lot of knowledge to work in the field of deafness, regardless of the patient's age group in the therapy, as well as the social and family context in which they are inserted.

B1: "We are very privileged to have our training, since we have a subject that will discuss family and a subject that will discuss deafness. We have a foundation that is to be able to work with it, so we have to remember that we are dealing with a family that has specific demands related to deafness, but it is a family, and there are some situations of spaces and times that all families will experience. And we still has to deal this issue of deafness, and we have a lot of support to work with these family issues, so our course deals very well with this question. The CEPRE works very well on these family issues, so this training is very important for us. We may get a little lost in the therapeutic practice, but the work with the family from the first year is very strong in our training, our work with parents. Our teachers always tell us that."

According to the students, the most necessary knowledge to work in therapy and that should be clear to the therapist, are the conceptions of deafness regarding the models and approaches that can be used with each deaf child/adolescent, according to their needs; problems and difficulties related to linguistic 
minority and deafness, regarding bilingualism and the education of the deaf person; the role of the family in the development of the deaf child, and the feelings that may arise during the development process of the individual and their family.

A2: "That's what we studied in the family (subject), right? The individual is in the mourning process, hence the acceptance and, therefore, each family will need a time for this. Sometimes, the person will stay a year in mourning, you know? The professional must understand this."

Considering the methods and approaches in education of deaf children/adolescents, the undergraduate students explained that the Brazilian Sign Language (BSL) course in the second year of the course introduced the basic concepts but, due to the small number of points for the subject, the program content was insufficient to meet all the demands brought by the deaf population in terms of mastery of a language. Thus, they reported that it would be important if the subject had a higher workload, since they believe that it would provide greater fluency to them.

For the development of the written language of deaf children/adolescents, the students emphasized the importance of visual resources to help them learn reading and writing. The students also emphasized that it is important to learn Portuguese as a second language to facilitate the communication of this child/ adolescent, since this will foster the socialization with the listening subjects.

It is also necessary to know the schooling process of the deaf individual to approach the inclusive processes in the therapy, as the use of Sign Language (BSL) in the social environment and the use of technologies and visual resources in the education of these subjects.

B2: "We are talking about an undergraduate course that should have BSL classes every semester if it was really focused on fluency, because a semester is really not enough. After all, how do you get fluent in English? You cannot get this in four semesters or even four years, so it's the same thing if you want to be fluent in Brazilian Sign Language. We will always have something missing, so since something is always going to be missing, then, unfortunately, or fortunately, we have to look for it. "

A3: "After all, we won't have a full conversation in $B S L$ with the child, since we are not fluent, and it's a language, so you have to be fluent."

B3: "I believe that the BSL subject should have a higher workload, as I did not have enough vocabulary, it was difficult for me to communicate with my patient and other patients of the practical training."

The students reported the importance of knowing the family with whom they are going to work, as well as developing actions to favor joint work, to have theoretical knowledge and practices applied in speech therapy and to have values such as sensitivity, empathy and patience.

\section{Expectations of students and families}

The students had the following expectations: the evolution of the deaf child/adolescent and the use of a common language (BSL). The students also highlighted the need to welcome the families of the deaf child/ adolescent in order to overcome the mourning for the loss of the idealized child and also so that they would engage in the care of the specifics related to their child. According to the students' perception, there is a lot of pressure on them from the family members, showing their expectations regarding the oralization of the deaf child/adolescent in many therapy sessions.

\begin{tabular}{|l|c|}
\hline Expectations of students and families with respect to deaf children/adolescents & Number of citations \\
\hline Family members with a common language (BSL) & 5 \\
\hline Experiencing grief & 7 \\
\hline Family members that understand, are patient and committed to care & 4 \\
\hline Joint work of family and the student & 3 \\
\hline Patient progress & 6 \\
\hline Understanding the importance of bilingualism and speech-language therapy & 2 \\
\hline
\end{tabular}

Figure 3. Expectations of students and families with respect to deaf children/adolescents 
The expectations of family members on knowing about the causes that lead to deafness and the particularities of it were also noticed. On the other hand, the students emphasized the importance of the family to understand the specificities of the speech-language pathology (care approaches in deafness, culture, deaf identity, hearing aids and implants) and the need to establish a common language with the deaf child/ adolescent and that family members have a psychological care, if necessary.

The main expectation of the students was that family members would be interested in learning the BSL, so that deaf children/adolescents would recognize themselves as a human being that has its place in space, with an identity and a culture.

Another expectation of the students was that family members would adopt bilingualism to establish a common language and that they essentially believe in the potential of their child and encourage other family members and close people to learn the BSL.

B1: "I hope they can, as long as necessary, understand the importance of bilingualism for a richer language acquisition for their children, as well as the importance of speech-language therapy to make it happen."

Students also hoped that, after understanding the importance of speech-language therapy, the family would collaborate with a work outside the clinic environment, making the social environment also a therapeutic and learning environment.

A2: "We expect families to help us to assist this child. We have an hour with this child, so when can't do much, as the family that stays with the child all the time. So we do all we can, but the family members are the ones who are going to practice everything we have done in therapy. We really hope that the family will put into practice everything we are trying to do and to show to the child."

B5: "They often expect a miracle from the speech-language pathologist and that the child will start right talking after therapy. It takes a lot of sensitivity to show the true role of the speech-language pathologist."

According to the G2, family members of patients should express their doubts and anxieties in order to enable a joint work with the professional. It is natural for the family to go through the process of dealing with a disabled child. The family has the stereotype of a perfect child, and when it does not meet expectations at birth, there is an intense process of mourning, which is a step that must be lived and overcome, until they reach acceptance and work to improve the quality of life of the deaf person.

B6: "Initially, upon receiving the diagnosis, parents are expected to go through a grieving process of the perfect child that they expected, in which each family deals in a way, and each family will have a different reaction. After this, parents will seek out the possibilities of their children to talk or to use only the BSL. Each family chooses the best way. It is hard to say what is expected from each one, but I expect several reactions, precisely because each family is different from the other. Some families may join therapy and participate in the whole process, while others may not get involved and stay more in distance. So the speech-language pathologist is responsible for integrating the family and knowing how to deal with each one."

According to the students, families have expectations at the beginning of the therapy that go beyond the simple assistance to the deaf child/adolescent and that refer to the possibilities of having greater insight about the evolution and possibilities of the deaf individual.

\section{Perception of undergraduate students on deaf children/adolescents}

Most students believe that both the deaf child/ adolescent and their families have specific needs, focusing mainly on the importance of the guidance on assistive technology resources for the deaf person, such as the cochlear implant and the PSAPs, as well as being aware of the importance of visual aids for deaf people. They also believe there is a need to welcome the family and to provide emotional and psychological support so that they can deal with the mourning of the idealized child.

B5: "They need someone to face the situation with them, showing the possibilities and potentials of their children. I believe so, because in many cases deafness affects the identity of the individual, their language and this may change the family dynamics and how they deal with the child, which requires a specific attention, taking into account their demands.

G1 believes that family members must know the diagnosis of the child/adolescent and understand deaf culture. 
A4: "Each disability is different from each other. Families of deaf people need several explanations on deafness, approaches, devices and implants. And that means that parents/family members may have the same questions for a long time, due to the need to know what works and how it works. Therefore, family members must be welcomed with patience, understanding that each one has their own time to deal with situations."

A7: "I believe they need to understand the deaf culture, how to stimulate the deaf child, what goes on with the child, the degree of deafness and the different therapeutic approaches. It is a complex issue to understand how the deaf person' interacts in the world, as they need focused and specific work, not being able to work with other disabilities alone, but also individual work."

According to the $\mathrm{G} 2$, it is essential that family members attend BSL classes and become fluent, in addition to making contact with deaf adults, so that they will be able to know and discover future perspectives for their children, such as work opportunities in the labor market and issues related to autonomy and independence. When they make contact with deaf adults, family members are able to encourage and motivate the deaf child/adolescent to carry out an activity in the future and to recognize the importance of the whole therapeutic process of knowledge and learning, which will be useful not only in the activities that they experience, but also for future skills.

B6: "There is a need to attend BSL classes, to receive guidance about hearing loss and PSAPS, that is, to really know and understand the diagnosis, you know? To understand that there is a culture of deaf community."

B2: "So, we have this work together with many professionals and also with parents, who have a psychologist, a deaf instructor, things related to the importance of seeing deaf adults. When parents arrive they often have that reaction: - But what will happen to the future of my child? And when you see a deaf adult person, it is very important to see their reaction: "- Look, he does this, and he does that, and take a look at it. So, it is very important to have a team to support, it is crucial to speech-language pathologists. We may not have all the resources; therefore we must have someone to work with, in order to help this family to see the possibilities."

\section{Bond and therapeutic practices}

Practices to improve the relationship between the student, the family member and the deaf child/ adolescent were emphasized as therapeutic practices that favor the bond. Results showed that 11 students sought ways to get closer to the family in order to favor the bond. Other actions were also reported as significant: to know the history and the clinical condition of the deaf child/adolescent and the preparation of the student with theoretical and practical contents for an adequate performance.

According to reports, family members have some difficulty in interacting with the deaf child/adolescent because they do not have a common language, because they feel that the child does not have the skills required to establish a conversation or because they do not accept the diagnosis. This fact may generate reflections on the evolution of the therapy. When family members understand the importance of treatment, the results of the evolution are visible.

The following practices were reported as practices that disfavored the bond: the school-clinic environment, the high number of absences of deaf children/adolescents, the lack of confidence of the families in the work with the student, and the absence of family members in the continuity of the speech-language pathology work at home.

According to $\mathrm{G} 1$, the evolution of therapeutic practices is impaired due to non-adherence of some deaf children/adolescents or their families or, due to the way in which the relationship between the therapist/ family member occurs, which consequently hinders the creation of a bond and the relation of the student with the patient, which is shown in Figure 4. 


\begin{tabular}{|c|c|c|c|}
\hline Practices that favored the bond & $\begin{array}{c}\text { Number of } \\
\text { citations }\end{array}$ & Practices that disfavored the bond & $\begin{array}{c}\text { Number of } \\
\text { citations }\end{array}$ \\
\hline $\begin{array}{c}\text { Knowing the patient's history and clinical } \\
\text { condition }\end{array}$ & 3 & Clinic-school environment & 3 \\
\hline Getting closer to the family members & 10 & High number of absences of patients & 5 \\
\hline Knowing the techniques used in therapy & 3 & Family members consulting the supervising \\
teacher first & 2 \\
\hline $\begin{array}{c}\text { Believing that you will make that the difference in } \\
\text { the therapeutic follow-up semester and working } \\
\text { for it }\end{array}$ & 2 & Only one semester of care for each student & 3 \\
\hline \begin{tabular}{c} 
Lack of trust in the care provided by the student \\
\hline
\end{tabular} & & Family not helping in the therapy & 6 \\
\hline
\end{tabular}

Figure 4. Perception of students on Bond and Therapeutic Practices

A1: "Interaction is of utmost importance for the good of the child and the family. So that the child feels welcomed by family members. Not all family members interact with their deaf relatives, it may be that they do not accept it, or because they do not feel comfortable with it and this must be worked, because it is also part of the therapeutic process."

According to G2, the difficulty of create bonds with family members is due to the fact that they sought the teachers responsible for the practical training when they have any doubts. Due to the clinic-school environment, the student only has six months with the child/ adolescent, while the supervising teacher is present all the time. Thus, when there is any doubt or when relatives need some information, they usually talk to the supervising teacher. Despite this perception, students reported that the adherence to the proposed therapy was not problematic, since the students considered the feedback and the evaluation of the teacher regarding their performance in the practical training.

B6: "In many times we talked and we developed aspects to be worked on (therapist and supervisor); however, as family members are users of the service (clinic-school) for a long time, sometimes they seeks other professionals to take their demands. It made me feel for a long time that there was no bond between us."

B3: "As for being a student, l've never felt any difference in expectations, at least not in the family. But the fact that I must change patients every semester does actually impair the therapy."

B1: "Since this patient's mother had been in the service for a long time, I felt that, when she had questions, she would sought out everyone first, and then she would tell me something, or sometimes her question would come to me through my supervisor: - A patient's mother came to talk to me in the hallway, and she's worried about these issues, can you work it out? So, I don't know if she does that because I'm a student, or because she knows the supervisor, since her child is in a very long process. Then as she already knew the supervisor from the beginning, I was the last person she was looking for and she was asking from others."

Some families see the student as the only alternative of improvement and they put all hopes in the therapy, which facilitates the development of the therapeutic practice.

B2: "We stay only for a short time, that is, we only have six months, so they are already used to have two therapists in the same year. Their bond is much stronger with the supervisor than with the therapist, and like it or not, they would turn to us and say: - You are so young, aren't you? And then, it' is up to us to show who we are. - Well, I'm young, but I studied to perform my work, l'm not here from nowhere, it was not a crazy thing out of my head. I have a theoretical reference that is not from just a young person, but from a person who also studied to be prepared."

Both groups of students recognized that the BSL is important for the dialogue with the deaf child/ adolescent and to the evolution of therapy; the bilingual approach is relevant for self-awareness of deaf culture and patient development.

B7: "You should be aware that there may be an unfulfilled expectation and you must accept that families need time, but you should also encourage the family to take action. It is all possible only with 
an educational background that addresses these issues (referring to bilingualism)."

For most students, there are several feelings to deal with the expectations of the family regarding the oralization of the deaf child/adolescent. The difficulty of seeing results due to the slow evolution of some cases causes anxiety and fear, and the obstacles in communication between the student and the child/ adolescent also impair the therapy, making the student feel incapable and highlighting the fear of providing care to this population due to the inexperience and to the claims of both the family and the practical training as a subject.

A1: "The practical training with deafness is a work that distresses me greatly, precisely due to the thorough evolution and to the difficulty of ways to try a more visible evolution."

B1: "Am I able to talk to that father? Of course, it scares a little, but when you get used to it, we already know what to do. So even if the patient looks at you from top to bottom and says: - Are you the one who is going to take care of my child? You already know how to deal and we can do it, we start to get used to conduct the conversation."

A3: "You still have the difficulty of working with others or working with the human aspect, with the pains of others and with all they brings to you. So, it is not always easy, and sometimes you can have years of experience and still get run out of action."

Both groups reported that they often do not find situations that they would regard as easy during the therapeutic process. The work is difficult at the beginning of the practical training, mainly due to the lack of experience.

B4: "I do not believe there is an easy situation, in my opinion this varies from individual to individual. However, the bond with the child makes it easy to reach the family, since it shows that we are together for the same purpose: to help the child. It requires much study, commitment, sensitivity, and even experience. This can all be acquired over time and therefore the practical training is just a 'taste' of it."

In order to improve the bond and therapy progress, G2 suggested that this practical training should last a year with the same deaf child/adolescent. G2 also suggested that some subjects of the course could go deeper on methods to apply in the practical training, so that students would not be required to learn everything too fast during practical training.

When listing the main feelings regarding the practical training of deafness, students highlighted anguish, frustration, inability and the fear of providing care to deaf children/adolescents. However, the students also presented the strengths and positive experiences over the practical training: respect for the progress of the deaf child/adolescent in the therapy, the empathy required for the success of it and the achievement and the pride after the conclusion of the therapy.

\section{DISCUSSION}

Data regarding the students' perceptions with respect to the supervised practical training in deafness show that they found difficulties when they started it in the fifth semester of the course because it was still unknown to them and due to the inexperience in the work with a deaf child/adolescent. In addition, they had to deal with their personal and academic expectations, as well as with the expectations of the family members of the deaf children/adolescents in order to achieve the success of the therapeutic project.

The relationship between theoretical and practical content was regarded as satisfactory and enabled them to act in the field of deafness, even with the challenges of being in a school clinic, presented by the family members of deaf children/adolescents in supporting the therapies, to understand the goals and accept that the students were the therapists. The students considered themselves privileged with respect to their academic formation.

A study with students of the Speech-Language Pathology course and whose purpose was the introduction and experience with the practice in hospital beds ${ }^{4}$ reported that the environment provided by the practical training brings additional concerns to curricular assessments, since students should contribute to the care of patients who deserve care, understanding and respect. In addition, there is an environment that can be stressful, both for the child/adolescent and for students and supervisors. There is an overload in dealing with the practical training, as a discipline that will evaluate students according to their performance, and the responsibility of providing therapeutic success, both to the patient and to their families ${ }^{4}$.

It is important to note that undergraduate students initiate their contact with patients and their families in this fifth semester, which may have highlighted the difficulties that they faced. 
Family, group dynamics and deafness subjects were considered the most important to students to work in the area. The BSL subject has been repeatedly mentioned as highly important, both for students and for the family members and patients; however, it provides a small amount of points, which generates a deficit in the work with the deaf person, thus hindering the communication.

The complexity of the curricular practical training appears when the student applies the theoretical knowledge in solving the demands brought by the patient in the care ${ }^{4}$. Implementing the acquired theoretical knowledge requires to understand the needs of the deaf child/adolescent for the clinical intervention. This is a challenge that is facilitated by the care sessions and by the consequent acquisition of practical experience.

The supervised training will be focused on training professionals with technical and political competence, providing knowledge, reasoning, perception and sensitivity for life and social issues, and to be able to act in contexts of uncertainty and complexity. Formal education is essential to the construction of the human being as a historical, political and social subject and, therefore, the supervised training is a place to share knowledge ${ }^{10}$.

The student also faces challenges in dealing with families and patients from different realities, which differ from their own and from a whole perspective that has been studied or constituted by social experience. In this way, it is necessary to recognize the importance and necessity of this process experienced by the student, since it prepares them to face a reality that is not shaped, but open to have a new form ${ }^{11}$.

Even with all the difficulties and pressures of the situation, they try and manage to deal with feelings and anguishes, which are often disabling and discourage them, thus reversing these feelings. It also makes them capable of providing a good care, which brings satisfaction to them and also to their patients, translating the appropriate use of competencies in the health area. Therefore, during their education, the student aims to get closer to the ideal, through the acquisition of skills that allow them to perform the care with perfection ${ }^{10}$.

To handle these situations, the student needs to learn to deal with feelings of transference and countertransference, which may influence their work as a therapist. The constant tension experienced by the students in relation to what has to be done to help the patients and what they can accomplish leads them to have the feelings mentioned, such as distress, anxiety and fear of providing care. The feelings of anxiety described by the students in the first contact with the patient compromise their performance, making it difficult or even blocking the action and the ability to effectively taking care of it.

Dealing with the family members of the deaf child/ adolescent was a heavily discussed point, both in the focal group and in completing the questionnaires. There is a specific pressure on the student who needs to be able to improve the condition as quickly as possible. The support of the family member is crucial for the good course of the therapeutical follow-up, because when the familiar one trusts the student, the kind of therapy and in approach adopted with regard to the case, he or she cooperates with the evolution of the therapeutical process, facilitating the bond. When the family believes in the effectiveness of the therapy and the possibility of results, the child does not lose the sessions. The bond created with the family and the patient helps the student to see the real difficulties of the child and what should be done to be improved.

The teacher is also an important factor during the practical training, both as knowledge sharer and as a safe haven during the training process. Some students in their initial experience of supervised curricular training may have a lack of confidence on technical procedures and in the relationship with the patient. The supervising teacher may assist in the achievement of self-confidence and the supervising teacher can assist in the attainment of self-reliance and confidence until they achieve professional empowerment. The student establishes an interpersonal relationship both with the patient and with the supervising teacher. It is believed that human relations are rich in meanings and contradictions and become a source of knowledge of the reality ${ }^{4}$.

The teacher may allow the students to learn together with the opportunity to work as a speech-language pathologist in the school clinic, which ensures a confidence in the development of their activities and a satisfaction by doing, in addition to the autonomy and responsibility towards the patient ${ }^{10}$.

Some students suggested that the fragmentation of the practical training in different times during the course does not favor the therapeutic follow-up. The supervised curricular training can be a clinically rich experience, but it is extremely punctual situation where students do not seek to identify their limitations and feelings due to the impossibility of a strong bond. The 
duration of the supervised deafness practical training is too short, since there are only 5 months of intervention with the patient, which sometimes leads to a negative impact of the performance in the improvement of the patient.

\section{CONCLUSION}

The analysis of the perception of undergraduate students in speech-language pathology about the therapy in the field of deafness showed that, initially, students only have theoretical knowledge and then difficulties arise due to lack of clinical knowledge, causing them to seek guidance of the teacher during supervision and also a connection with the theoretical content already studied. The experience gained along the stage provided theoretical-practical knowledge to work with the family, recognizing the importance of this to the therapeutic success and to deal with the expectations of both parties. Dealing with deafness with the aim of encouraging expression and communication is not an easy task, but the difficulties have been worked and overcome according to the acquisition of new experiences, family support and the creation of a bond between the student, the child/adolescent and their family members.

\section{REFERENCES}

1. Conselho Federal de Fonoaudiologia. Resolução CNE/CES 5, de 19 de fevereiro de 2002. Institui Diretrizes Curriculares Nacionais do Curso de Graduação em Fonoaudiologia. Disponível em: http://www.fonoaudiologia.org.br/cffa/wp-content/ uploads/2013/07/dc.pdf

2. Goldberg K, Kieling ML, Guzzo F. Centro de psicologia aplicada da uri-campus de Erechim: da concepção à construção. Rev. Ciênc. Hum. 2012;9(12):125-40.

3. Peixoto LF, Celeste LC, Silva EM, Mangili LD. Quality assessment/satisfaction of the learning of practical discipline of the Speech, Language and Hearing Sciences course. Distúrb. Comun. 2017;29(4):625-35.

4. Queiroz MAS, Teixeira CLV, Braga CM, Almeida KA, Pessoa RX, Almeida RCA et al. Curricular supervised traineeship: perceptions of student-therapist in Speech, Language and Hearing Sciences in a hospital. Rev. CEFAC. 2013;15(1):135-43.

5. Isotani SM, Ávila CRB, Puccini RF. Curricular changes in the undergraduate degree in Speech,
Language and Hearing Sciences from UNIFESP. Distúrb. Comun. 2017;29(2):237-50.

6. Moraes BA, Costa NMSC. Compreendendo os currículos à luz dos norteadores da formação em saúde no Brasil. Rev. Esc. Enferm. USP. 2016;50(especial):9-16.

7. Souza DMB, Lopes SMB. The family's perception of speech therapy in an outpatient unit. Rev. CEFAC. 2015;17(1):80-7.

8. Minayo MCS. Análise qualitativa: teoria, passos e fidedignidade. Ciênc. Saúde Coletiva. 2012;17(3):621-6.

9. Sehnem GD, Alves CN, Wilhelm LA, Ressel LB. Focal group utilization as data gathering technic to researches: experience report. Ciênc. Cuid. Saúde. 2015;14(2):1194-200.

10. Lima TC, Paixão FRC, Cândido EC, Campos CJG, Ceolim MF. Supervised curricular internship: analysis of the students' experience. Rev Bras Enferm. 2014;67(1):133-40.

11. Benito GAV, Tristão KM, Paula ACSF, Santos MA, Ataíde LJ, Lima RCD. Developing of general competencies during supervised clinical practice. Rev Bras Enferm. 2012;65(1):172-8. 